\title{
Erratum to: Engage Your Nurses, Clinicians and Patients to Drive Your Hospital
}

\author{
Erratum to: \\ Chapter 1 (page 7) in: E. Weimann, P. Weimann, High Performance in Hospital \\ Management, DOI 10.1007/978-3-662-49660-2_1
}

A zero went missing regarding the mortality rate (100 instead of 1000) on page 7, under heading 1.3.2 South African Health System as a Prototype for Developing Countries in chapter 1 . The corrected paragraph is given below.

\subsubsection{South African Health System as a Model for Developing Countries}

The South African health system is characterised by a severe divide between the public and private sectors. The proposed NHI aims to bridge the existing health inequalities and offer equal access to affordable, quality health care to all citizens, irrespective of their socioeconomic status (Frogner 2010). The South African health system is characterised not only by a two-tiered system, but also by escalating costs. Further, while the costs in the private health sector almost doubled between 1996 and 2003, spending in the public sector decreased. Annual expenditure per capita on private care is estimated to be four times higher than in the public sector. In addition, a major part of public health sector spending is directed towards HIV/AIDS and TB treatment, to the neglect of other medical areas (Coovadia et al. 2009).

The updated online version of this chapter can be found at DOI 10.1007/978-3-662-49660-2_1 
Adding to the decline in the quality of public health services are the poor governance and management of hospitals, public underfunding, mismanagement, shortages of health professionals and deteriorating infrastructure (Keeton 2010). South Africa needs to invest in the training of health professionals: this is currently underdeveloped, indeed it is neglected. The use of measures that optimise efficiency and enable the treatment of patients according to their needs such as the triage score, are also proposed for the country. The escalating gap between the rich and the poor in South Africa is underlined by the increasing Gini index over the last decade (59.0 in 1993 and 65.0 in 2009), which indicates that the disparity is wider than under apartheid. The country spends $8.9 \%$ of its gross domestic product (GDP) on its health care system, with a poor outcome that is emphasised by a low life expectancy (57 years in men versus 60 years in women) and a high neonatal mortality rate (19 per 1000 live births in 2011). Most financial and human resources in the health care sector are currently located in the private health sector, which covers only a relatively wealthy minority of the population. 